\title{
Impact of intrapartum antimicrobial prophylaxis upon the intestinal microbiota and the prevalence of antibiotic resistance genes in vaginally delivered full-term neonates
}

Alicja Nogacka ${ }^{1}$ N Nuria Salazar ${ }^{1}$, Marta Suárez², Christian Milani ${ }^{3}$ Silvia Arboleya ${ }^{1,4}$, Gonzalo Solís², Nuria Fernández², Lidia Alaez ${ }^{1}$, Ana M. Hernández-Barranco ${ }^{1}$, Clara G. de los Reyes-Gavilán' ${ }^{1}$, Marco Ventura $^{3}$ and

Miguel Gueimonde ${ }^{1^{*}}$ (D)

\begin{abstract}
Background: Disturbances in the early establishment of the intestinal microbiota may produce important implications for the infant's health and for the risk of disease later on. Different perinatal conditions may be affecting the development of the gut microbiota. Some of them, such as delivery mode or feeding habits, have been extensively assessed whereas others remain to be studied, being critical to identify their impact on the microbiota and, if any, to minimize it. Antibiotics are among the drugs most frequently used in early life, the use of intrapartum antimicrobial prophylaxis (IAP), present in over 30\% of deliveries, being the most frequent source of exposure. However, our knowledge on the effects of IAP on the microbiota establishment is still limited. The aim of the present work was to evaluate the impact of IAP investigating a cohort of 40 full-term vaginally delivered infants born after an uncomplicated pregnancy, 18 of which were born from mothers receiving IAP.

Results: Fecal samples were collected at 2, 10, 30, and 90 days of age. We analyzed the composition of the fecal microbiota during the first 3 months of life by 165 rRNA gene sequencing and quantified fecal short chain fatty acids by gas chromatography. The presence of genes for resistance to antibiotics was determined by PCR in the samples from 1 -month-old infants. Our results showed an altered pattern of intestinal microbiota establishment in IAP infants during the first weeks of life, with lower relative proportions of Actinobacteria and Bacteroidetes and increased of Preoteobacteria and Firmicutes. A delay in the increase on the levels of acetate was observed in IAP infants. The analyses of specific antibiotic resistance genes showed a higher occurrence of some $\beta$-lactamase coding genes in infants whose mothers received IAP.

Conclusions: Our results indicate an effect of IAP on the establishing early microbiota during the first months of life, which represent a key moment for the development of the microbiota-induced host homeostasis. Understanding the impact of IAP in the gut microbiota development is essential for developing treatments to minimize it, favoring a proper gut microbiota development in IAP-exposed neonates.
\end{abstract}

Keywords: Intestinal microbiota, Microbiome, Antibiotics, Neonate, Intrapartum antimicrobial prophylaxis, Antibiotics

\footnotetext{
* Correspondence: mgueimonde@ipla.csic.es

${ }^{1}$ Department of Microbiology and Biochemistry of Dairy Products, Instituto

de Productos Lácteos de Asturias. Consejo Superior de Investigaciones

Científicas (IPLA-CSIC), Ctra. Infiesto s/n, 33300 Villaviciosa, Asturias, Spain

Full list of author information is available at the end of the article
} 


\section{Background}

The human gut harbors a rich and complex microbiota whose establishment begins in early life. This microbiota contributes to an appropriate gut development and intestinal barrier function, resulting essential for the metabolic and immune homeostasis of the host [1, 2]. During the last years, an increasing amount of scientific evidence has underlined the important role of the early-life gut microbiota establishment and the development of such microbiota, in the later health of the individual [2-6]. This early microbiota-host interaction affects not just the local gut environment but also distal organs [7, 8]. Therefore, any disturbance on the intestinal microbiota development process in these early moments may have important implications for the infant's health and also for the risk of disease later on life. Indeed, an aberrant early microbiota has been found to precede the development of diseases [9-11].

Traditionally, infants have been considered sterile in utero, but recent data support the occurrence of a certain degree of microbial exposure prior to delivery [12]. At birth, the microbial exposure becomes massive and the newborn is rapidly and densely populated by a complex microbiota. This microbial colonization begins with facultative anaerobic and aerotolerant microorganisms which reduce the intestinal environment allowing the further establishment of strict anaerobes, such as Bifidobacterium and Bacteroides [13, 14]. After the initial colonization steps, the infant microbiota often appears dominated by Actinobacteria, frequently accompanied also by high levels of Proteobacteria [15-17]. This intestinal colonization process is going to be dependent on both genetic and environmental factors [18]. Among the last ones, many different prenatal and postnatal conditions may be affecting the colonization process, including gestational age at birth, mode of delivery, use of antibiotics, or feeding habits [17-21]. Some of these factors, such as being born by $\mathrm{C}$-section or receiving antibiotics, have been associated with an increase in the risk of later disease [22].

Antibiotics are among the drugs most frequently used in early life. During the last years, different animal studies have demonstrated that reduced exposure to microbes early in life is associated with an increased risk of later disease [11, 23]. Moreover, antibiotics-induced microbiota dysbiosis in early life leads to increased susceptibility to allergic and metabolic disorders [24-26]. The most frequent cause of exposure to antibiotics during the perinatal period is the use of intrapartum antimicrobial prophylaxis (IAP), which is present in over $30 \%$ of deliveries [27]. Several studies have shown that early postnatal antibiotic exposure disturbs the natural establishment of the intestinal microbiota in the newborn with potential negative influence in later health $[28,29]$. However, with the exception of some preliminary culture-based assessment [30], only recently studies focusing on the impact of IAP on the microbiota development, in both preterm and full-term newborns, have started to be available [17, 31-33]. In spite of this, our knowledge on the effects of IAP on the intestinal microbiota development is still limited.

The aim of the present study was to evaluate the impact of IAP on the establishment of the gut microbiota in the newborn. To avoid the presence of potential confounders, we focused on vaginally delivered full-term infants remaining healthy during the length of the study. To this end, we used 16S rRNA gene sequence-based fecal microbiota analysis, gas chromatography (GC) for quantification of fecal short chain fatty acids (SCFAs) as a measure of microbiota metabolic activity, and PCR for the detection of specific antibiotic resistance genes.

\section{Methods}

\section{Study participants}

The study was approved by the Regional Ethical Committee of Asturias Public Health Service (SESPA), and an informed written consent was obtained from each infant's parents. The study included 40 full-term (gestational ages $>37$ weeks) vaginally delivered infants born after an uncomplicated pregnancy at the Central University Hospital of Asturias (Northern Spain). Eighteen of the mothers received intrapartum antimicrobial prophylaxis (in all cases, an initial dose of 5 million units of penicillin followed by 2.5 million units every $4 \mathrm{~h}$ until delivery; in most cases, the mothers received three or less doses), due to confirmed or suspected vaginal colonization by group-B-streptococci (IAP group); whilst, the other 22 mothers, all of them being culture negative for group-B-streptococci, were not exposed to antibiotics (no-IAP group). None of the mothers received antibiotics during pregnancy or the postnatal period, other than the above mentioned IAP, and none of the infants received antibiotics during the duration of the study. Eighteen infants from the no-IAP and 11 from the IAP group were exclusively breast-fed during the study period whilst the remaining newborns (four in the no-IAP and seven in IAP group; difference no statistically significant) received infant formula milk. All infants were discharged from the hospital after 2 or 3 days of life.

\section{Sample collection}

Fecal samples were collected at 2, 10, 30, and 90 days of age. A fresh fecal sample was taken in a sterile container by the parents and immediately frozen at $-20{ }^{\circ} \mathrm{C}$. Samples were sent within 1 week to the laboratory where they were stored at $-80{ }^{\circ} \mathrm{C}$ until DNA extraction.

\section{Fecal DNA extraction}

Fecal samples were thawed, weighed, diluted 1/10 in sterile PBS solution, homogenized in a LabBlender 400 stomacher for $5 \mathrm{~min}$, and DNA was extracted using the 
QIAamp DNA stool kit (Qiagen GmbH, Hilden, Germany) as previously described [14]. Extracted DNA was kept frozen at $-80{ }^{\circ} \mathrm{C}$ until analysis.

\section{S rRNA gene sequence-based microbiota analysis}

Partial 16S rRNA gene sequences were amplified from extracted DNA using primer pair Probio_Uni and /Probio_Rev, which target the V3 region of the $16 \mathrm{~S}$ rRNA gene sequence [34]. 16S rRNA gene sequencing was performed using a MiSeq (Illumina) according to the protocol previously reported [34]. Following sequencing, the obtained individual sequence reads were filtered by the Illumina software to remove low quality sequences. All Illumina quality-approved, trimmed, and filtered data were exported as .fastq files. The fastq files were processed using a custom bash script based on the QIIME software suite [35]. This script performs a quality control of the sequences and retains only those with a length between 140 and $400 \mathrm{bp}$ as well as mean sequence quality score $>20$, truncates sequences at the first base if a low quality rolling $10 \mathrm{bp}$ window was found, and removes reads with homopolymers $>7$ bp as well as with mismatched primers. The custom bash script then integrates Qiime python scripts in order to generate de novo 16S rRNA Operational Taxonomic Units (OTUs) with $\geq 97 \%$ identity using uclust [36], remove OTUs with less than 10 sequences, pick a random reference sequence for each OTUs, classify OTUs down to the genus level by means of the SILVA database v.123 [37], remove chimeric sequences using ChimeraSlayer (http:// microbiomeutil.sourceforge.net/), and calculate alphadiversity rarefaction curves using chao1, Shannon, and observed number of OTUs indices.

\section{SCFAs analysis}

The concentration of SCFAs in feces $(\mathrm{mM})$ was determined in a chromatographic system composed of two 6890 N GC (Agilent Technologies Inc., Palo Alto, CA, USA) connected to a FID and a MS 5973N detector as previously described [38].

\section{Detection of antibiotic resistance genes in fecal samples}

The occurrence of different antibiotic resistance genes in fecal samples from 1-month-old infants was determined by PCR (UnoCycler, VWR International, Pennsylvania, USA) using previously described primers (Table 1). All oligonucleotides were purchased from Macrogen Europe (Macrogen, Amsterdam, The Netherlands). PCRs were performed in a total volume of $25 \mu \mathrm{l}$ containing $1 \mu \mathrm{l}$ of fecal DNA extract as a template. The reaction mixture was composed of $1 \times$ Dream PCR Master Mix (Thermo Fischer Scientific, San Jose, CA, USA) using $0.2 \mu \mathrm{M}$ of each primer. The thermal cycle program consisted of the following time and temperature profile: an initial cycle of $94{ }^{\circ} \mathrm{C}$ for $5 \mathrm{~min}, 35$ cycles of $30 \mathrm{~s}$ at $94{ }^{\circ} \mathrm{C}, 30 \mathrm{~s}$ at the annealing temperature of the corresponding primer pair (Table 1) and $1 \mathrm{~min}$ at $72{ }^{\circ} \mathrm{C}$, and a final extension step of $7 \mathrm{~min}$ at $72{ }^{\circ} \mathrm{C}$. Amplified products were subjected to gel electrophoresis in 1\% agarose gels and were visualized by ethidium bromide staining.

\section{Statistical analyses}

Results were analyzed using the SPSS software (SPSS Inc. Chicago, USA). The normality of the data, at each sampling point, was checked, and some of the bacterial groups showed non-normal distribution; therefore, differences

Table 1 Primers and annealing temperatures used for the detection of antibiotic resistance genes by PCR

\begin{tabular}{|c|c|c|c|c|c|}
\hline Gene & Oligonucleotide & $\begin{array}{l}\text { Annealing } \\
\mathrm{T}^{\circ}\end{array}$ & Antibiotic group & Resistance mechanisms & Ref. \\
\hline $\operatorname{tet}(\mathrm{W})$ & $\begin{array}{l}\text { F-AAGCGGCAGTCACTTCCTTCCR- } \\
\text { TCAAGTATCCCAGCGAAACC }\end{array}$ & 60 & Tetracyclines & Ribosomal Protection Protein & [49] \\
\hline $\operatorname{tet}(\mathrm{M})$ & $\begin{array}{l}\text { F-ACAGAAAGCTTATTATATAACR- } \\
\text { TGGCGTGTCTATGATGTTCAC }\end{array}$ & 55 & Tetracyclines & Ribosomal Protection Protein & [49] \\
\hline $\operatorname{tet}(O)$ & $\begin{array}{l}\text { F-ACGGARAGTTTATTGTATACCR- } \\
\text { TGGCGTATCTATAATGTTGAC }\end{array}$ & 60 & Tetracyclines & Ribosomal Protection Protein & [49] \\
\hline $\operatorname{tet} A(B)$ & F-TTGGTTAGGGGCAAGTTTTGR-GTAATGGGCCAATAACACCG & 55 & Tetracyclines & Efflux pump & [63] \\
\hline$b l a_{\text {tem }}$ & F-TTTCGTGTCGCCCTTATTCCR-CCGGCTCCAGATTTATCAGC & 60 & Penicillins & $\beta$-lactamase & [63] \\
\hline bla & $\begin{array}{l}\text { F-ATGTGCAGYACCAGTAARGTKATGGCR- } \\
\text { GGGTRAARTARGTSACCAGAAYSAGCGG }\end{array}$ & 60 & Penicillins & $\beta$-lactamase & {$[50]$} \\
\hline$b l a_{\mathrm{SHV}}$ & F-CACTCAAGGATGTATTGTGR-TTAGCGTTGCCAGTGCTCG & 58 & Penicillins & $\beta$-lactamase & [64] \\
\hline mecA & $\begin{array}{l}\text { F-GGGATCATAGCGTCATTATTCR- } \\
\text { AACGATTGTGACACGATAGCC }\end{array}$ & 56 & Penicillins & Penicillin-binding protein $2 \mathrm{a}$ & {$[50]$} \\
\hline $\begin{array}{l}\operatorname{aac}\left(6^{\prime \prime}\right)-1 e- \\
\operatorname{aph}\left(2^{\prime \prime}\right)\end{array}$ & $\begin{array}{l}\text { F-CCAAGAGCAATAAGGGCATACCR- } \\
\text { CACACTATCATAACCATCACCG }\end{array}$ & 55 & Aminoglycosides & $\begin{array}{l}\text { Bifunctional acetyltransferase } \\
\text { phosphotransferase }\end{array}$ & [64] \\
\hline strA & F-CTTGGTGATAACGGCAATTCR-CCAATCGCAGATAGAAGGC & 65 & Aminoglycosides & Phosphotransferase & [63] \\
\hline $\mathrm{cm} / \mathrm{A} 1$ & F-CACCAATCATGACCAAGR-GGCATCACTCGGCATGGACATG & 60 & Chloramphenicol & Efflux pump & [63] \\
\hline
\end{tabular}


between groups of infants were analyzed using nonparametric tests (Mann-Whitney $U$ test or Kruskal-Wallis test). Pearson $\chi^{2}$ test and logistic regression were used for assessing the occurrence of the different antibiotic resistance genes analyzed in both groups.

\section{Nucleotide sequence accession numbers}

The raw sequences reported in this article have been deposited in the NCBI Short Read Archive (SRA) under accession number PRJNA362530.

\section{Results}

Impact of IAP on the intestinal microbiota composition of the newborn

Sequencing of the PCR products obtained by amplification of the V3-V4 region of the 16S rRNA gene from the samples analyzed yielded an average of $\sim 60,000$ filtered partial sequences per sample with an average length of $178 \mathrm{bp}$. A minimum of 47,000 sequences per sample were used for standardizing the microbiota measures. Rarefaction curves indicated that the sequencing depth was enough since the samples reached the plateau phase (Additional file 1: Figure S1). IAP was found to reduce alpha-diversity in comparison with no-IAP infants (Additional file 2: Figure S2).

The microbiota of our full-term vaginally delivered infants was initially (2 days of age) dominated by Proteobacteria which represented a relative proportion of $67 \%$ in infants from mothers receiving IAP and of $50 \%$ in non-IAP-exposed infants (Fig. 1). The levels of this bacterial phylum decreased along time reaching levels of $46 \%$ in IAP-exposed and $35 \%$ in non-exposed infants at 10 days of age. These levels were further reduced to 36 and $27 \%$, respectively, at 1 month of age and 34 and $32 \%$ at the age of 3 months. In spite of these general lower levels of Proteobacteria in non-IAP-exposed babies, compared with infants from IAP-receiving mothers, the differences did not reach statistical significance $(p>0.05)$, likely due to the high inter-individual variation. The level of Firmicutes in IAP infants increased from an initial $24 \%$ at 2 days of age to a $38 \%$ at 10 days, the levels remaining stable afterwards. In contrast, the levels of this microbial phylum did not show this increase and were maintained constant in the control group (infants not exposed to IAP), with only minor variations along time (Fig. 1). This different behavior of Firmicutes in IAP vs. control infants resulted in significantly higher levels $(p<0.05)$ of this phylum in the former group at 10 and 90 days of age. On the contrary to that observed for Proteobacteria and Firmicutes, the relative proportions of Actinobacteria, Bacteroidetes, and others were higher in control than in IAP-exposed infants (Fig. 1). These differences reached statistical significance at 10 days of age for Actinobacteria.

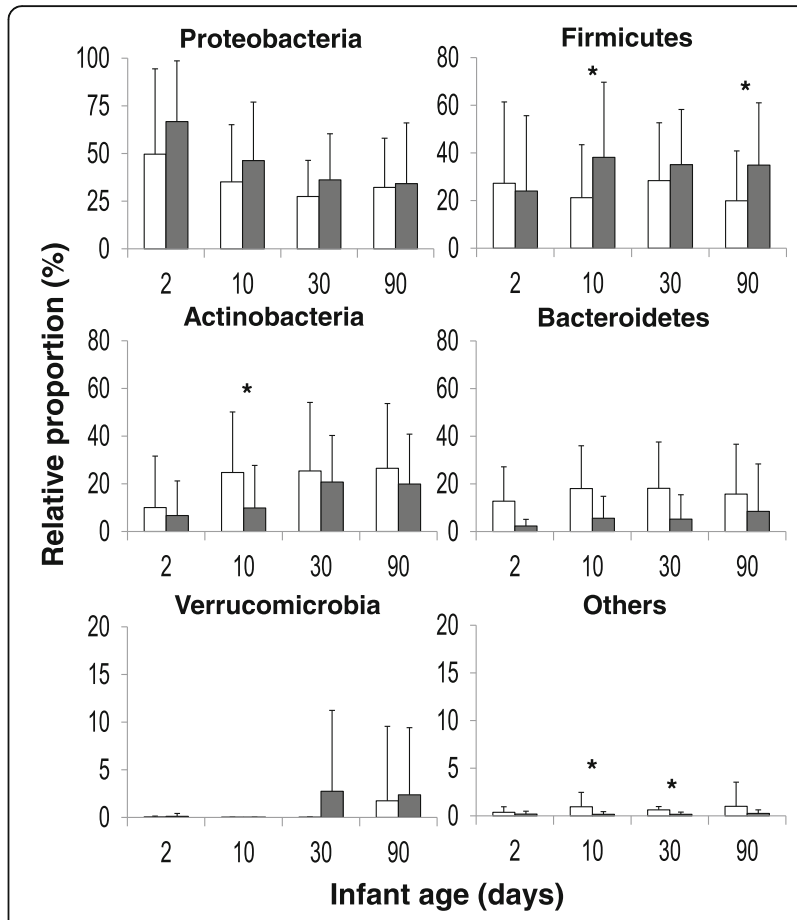

Fig. 1 Relative proportions (\%) of the main bacterial phyla present in the fecal samples obtained from both infant groups, newborns whose mothers received IAP (black columns) and those whose mothers dis not (white columns), at the different sampling points. Asterisk indicates statistically significant differences $(p<0.05)$ between both groups of infants

Analyses of the data at family level confirmed these differences, with some families showing statistically significant changes related to the use of IAP (Additional file 3: Table S1). Among these, it is important to underline the significantly lower levels of Bifidobacteriaceae and unclassified Actinobacteria $(p<0.05)$ found in IAP infants. On the contrary, the latter group of infants presented increased levels $(p<0.05)$ of Prevotellaceae at 2 and 90 days of age, Rikenellaceae at 2 days of age, Clostridiaceae at 10 days of age, and of Campylobacteriaceae and Helicobacteriaceae at the end of the study (3 months). Moreover, the levels of the group classified as Family S24-7 (Muribaculaceae), a Bacteroidetes group commonly found in feces from homoeothermic animals [39], but whose first cultured representative remained elusive till recently [40], were significantly increased in IAP infants all along the study.

Impact of IAP on the intestinal production of SCFAs in the infant gut

The levels of the main fecal SCFAs (acetate, propionate, and butyrate) increased over time in both infant groups. Infants whose mothers received IAP showed a trend towards lower levels of propionate and acetate during the first days of life (Fig. 2). These differences were statistically 


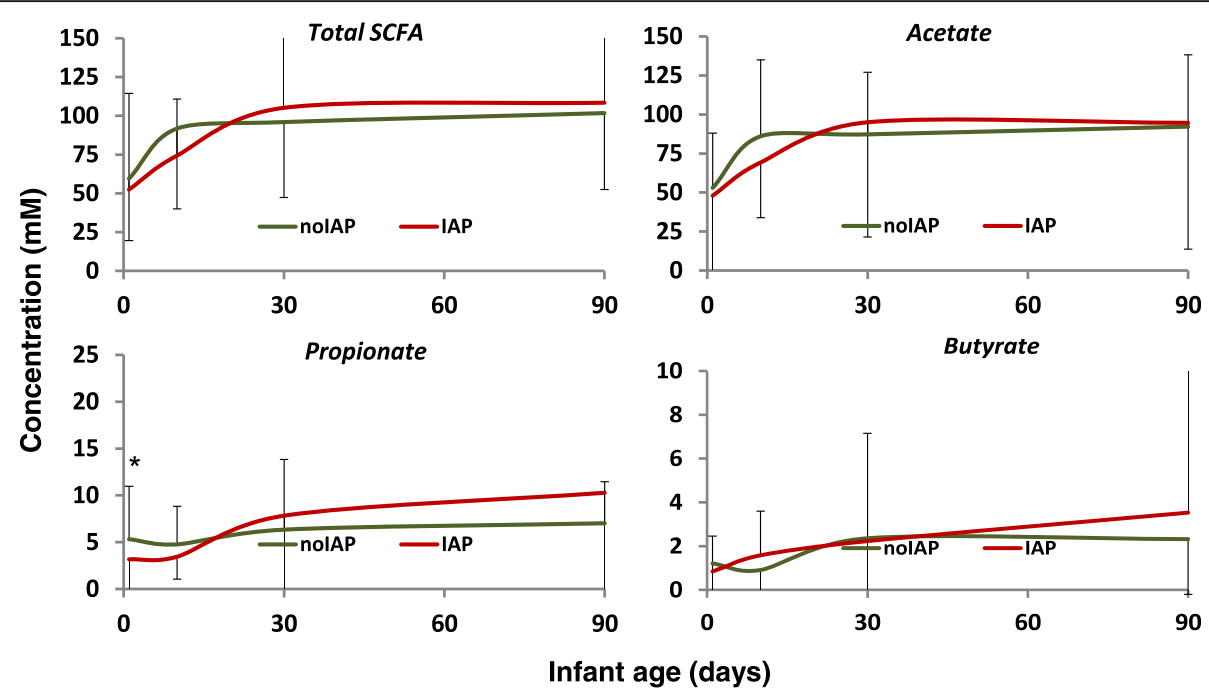

Fig. 2 Concentration (mM) of acetate, propionate, and butyrate and total SCFAs in feces from infants whose mothers received IAP (IAP; $n=18$ ) and those whose mothers did not (nolAP; $n=22$ )

significant $(p<0.05)$ for propionate at 2 days of life, when the levels of these SCFAs in infants not exposed to IAP almost doubled those in IAP infants $(5.3 \mathrm{vs} 3.1 \mathrm{mM}$, respectively). However, likely due to the high interindividual variability observed in the levels of fecal SCFAs, no other statistically significant differences were obtained. This apparent delay in the production of acetate and propionate during the first days of life disappeared already at 30 days of age, when the levels of SCFAs in IAP infants were even slightly higher $(p>0.05)$ than in the control infants, and remained mostly unchanged during the rest of the study. When the SCFAs were calculated as relative proportions (percentage with regard to the total SCFAs level), no statistically significant differences were observed at any time point analyzed (Additional file 4: Figure S3).

\section{Effect of feeding habits on the impact of IAP upon the infant intestinal microbiota composition}

To assess the potential influence of the infant feeding habits upon the observed effects of IAP on the establishing infant gut microbiota, we compared the data obtained for the exclusively breast-fed babies with those for formula-fed ones, both in mothers receiving and not receiving IAP. The results showed differences in the microbiota depending on the infant feeding habits as well as differential responses to IAP between both infant groups (Additional file 5: Table S2). Exclusively breastfed infants not exposed to IAP showed higher levels of Bacteroidetes all along the study and lower early levels of Proteobacteria than unexposed formula-fed ones. Interestingly, regardless of IAP exposure, lower levels of Actinobacteria were observed in formula-fed newborns during early days life, but this trend changed afterwards with formula-fed infants showing higher levels after
1 month of age. Similarly, Verrucomicrobia levels were higher in the formula-fed group at the end of the study (90 days of age).

Regarding the response to IAP, antibiotics exposure reduced the levels of Actinobacteria and increased those of Firmicutes, and to a lesser extent Proteobacteria, in both breast and formula-fed infants (Additional file 5: Table S2). In the case of Bacteroidetes the response to IAP was variable depending on the infant feeding pattern; thus whereas IAP reduced the levels of this phylum in exclusively breast-fed infants, the contrary was true in formula-fed ones. To further delineate this differential behavior, the relationship between non-IAP and IAP group was calculated for the different microorganisms in both breast-fed and formula-fed infants (Fig. 3). As expected from the composition data, the levels of Proteobacteria and Firmicutes were higher in IAPexposed infants rendering a low ratio. This was true for both breast- and formula-fed infants. However, in the case of Actinobacteria and to a larger extent of Bacteroidetes, the impact of IAP seems to be different between breast-fed and formula-fed babies (Fig. 3). This is especially true for Bacteroidetes since all the main representative families of this phylum (Bacteroidaceae, Prophyromonadaceae, and Prevotellaceae) showed a lower ratio between non-IAP and IAP babies in the case of the formula-fed newborns (Fig. 3). Similar results were also obtained for the families P5D1-392, including unculturable microorganism from the Lactobacillales order, Lachnospiraceae, Ruminococcaceae, or Acidaminococcaceae, among others (Fig. 3).

\section{Impact of IAP on the carriage of antibiotic resistance genes}

The assessment of the occurrence of different antibiotic resistance genes in the feces of 1-month-old infants by 


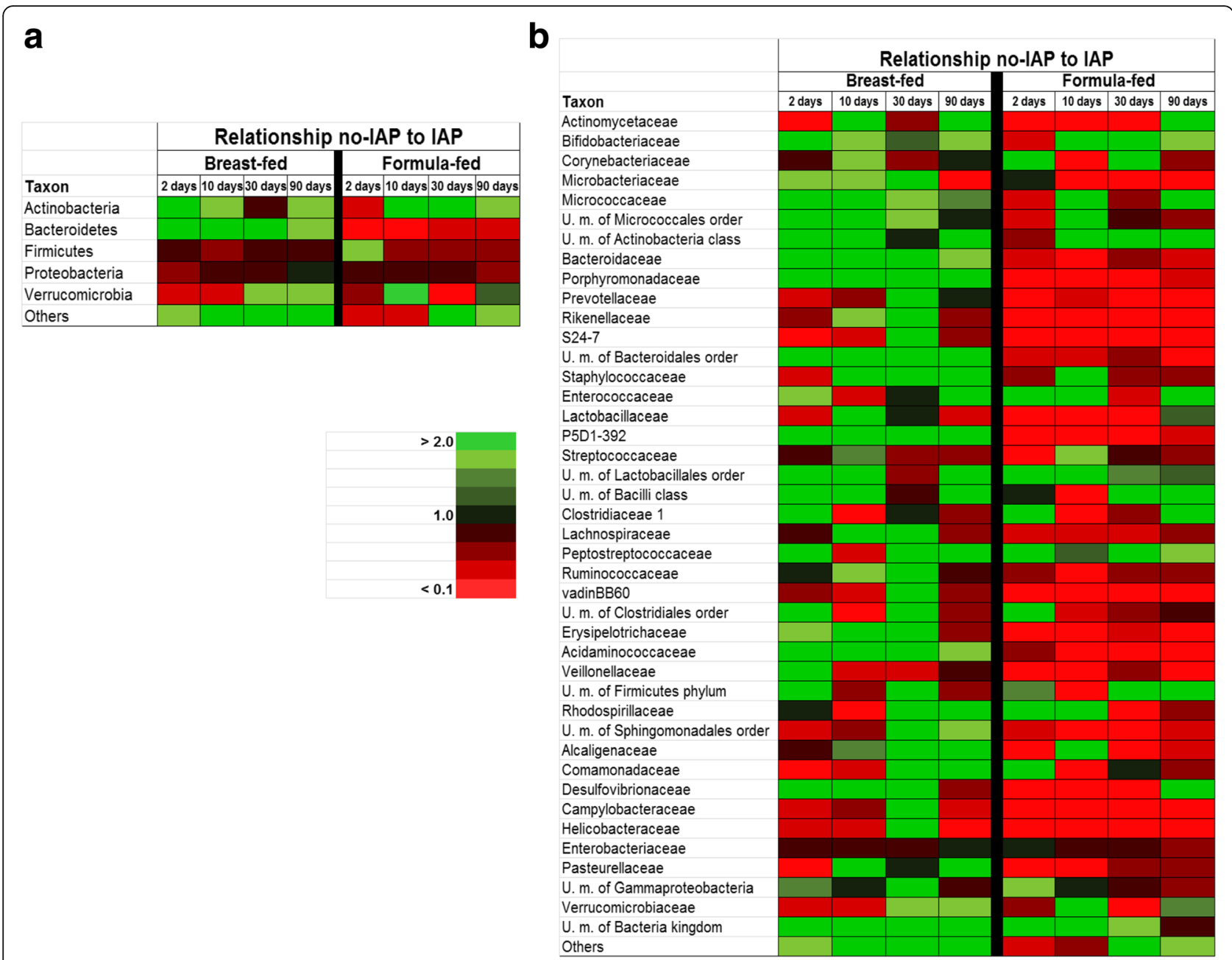

Fig. 3 Ratio obtained for the different microbial phyla $(\mathbf{a})$ and families (b) between no-IAP exposed infants (babies whose mothers did not receive IAP) and IAP-exposed ones in exclusively breast-fed infants ( $n=29,10$ no-IAP and 11 IAP) and in formula-fed ones ( $n=11,2$ no-IAP and 7 IAP). The ratio was calculated as relative proportion in no-IAP/relative proportion in IAP newborns at the different time points analyzed

PCR with specific primer pairs showed variability among the analyzed genes (Table 2). Whilst, the genes tet $\mathrm{M}$, tet $\mathrm{O}$, tet $\mathrm{A}$, strA, or $\operatorname{clm} \mathrm{A} 1$ were not detected in any sample; others such as $B L A$-tem, mecA, or $C T X-\mathrm{M}$ resulted positive in more than $30 \%$ of the infants. Interestingly, the $\beta$-lactamase genes $B L A$-tem, $C T X-M$, and the gene acc6-aph2, conferring resistance to aminoglycosides,

Table 2 Occurrence (\% of positive samples) of the different antibiotic resistance genes analyzed, in fecal samples from 1-month-old infants from mothers receiving IAP $(n=18)$ and those from mothers that did not receive intrapartum antibiotics ( $n=22)$

\begin{tabular}{lllllll}
\hline Group & \multicolumn{6}{l}{ Antibiotic resistance gene $^{\text {a }}$} \\
\cline { 2 - 7 } & tetW & BLA-tem & mecA & CTX-M & BLA-shv & acc6-aph2 \\
\hline No-IAP & $9.0 \%$ & $61.9 \%$ & $57.1 \%$ & $33.3 \%$ & $14.3 \%$ & $23.8 \%$ \\
IAP & $7.7 \%$ & $82.3 \%$ & $44.4 \%$ & $52.9 \%$ & $11.1 \%$ & $38.9 \%$ \\
\hline
\end{tabular}

${ }^{\mathrm{a}}$ None of the samples was positive for tetM, tetO, tetA, strA, or $\mathrm{cm} / \mathrm{A} 1$ showed an increased occurrence in infants from IAPreceiving mothers than in non-antibiotic exposed ones (Table 2). However, these differences did not reach statistical significance $(p>0.05)$; although in the case of BLA-tem, a trend towards increased occurrence seems to be present $\left(x^{2} ; 0.05<p<0.01\right)$, which is in agreement with the logistic regression analyses that also suggest IAP as a predictor of the occurrence of this gene (Odds ratio 4.3; $p<0.1$ ).

\section{Discussion}

During the last decade, we have started to understand the critical role of the early-life microbiota-host interaction as a determinant of later health $[2,41]$. To this regard, the use of antibiotics may influence gut microbiota composition with consequences for later health [42]. Moreover, incomplete microbiota recovery after antibiotics administration, in both adults and infants, has been observed $[43,44]$. These results point out to the need of 
defining and understanding the factors, such as IAP, which may determine the initial establishment of the gut microbiota.

In general, the composition of the gut microbiota in our study is similar to that reported by other authors in other full-term vaginally delivered baby cohorts $[17,32,34,44$, 45]. As it is common in this typology of studies, a large inter-individual variability was observed, which hampers the statistical analyses of the results. Despite this large variability, our results demonstrate the impact of maternal IAP upon the composition of the gut microbiota in the infant during the first months of life. Similarly to other recent 16S rRNA gene-based studies, carried out in both preterm and full-term infants [17,31-33], we observed a reduction on the levels of Actinobacteria and Bacteroidetes and an increase of Proteobacteria and Firmicutes in babies from mothers receiving IAP than in those not exposed to antibiotics. To note, infants in the IAP group showed reduced proportions of commensal microorganisms, such as the family Bifidobacteriaceae, but increased of potentially pathogenic microorganisms including Campylobacteriaceae or Helicobacteriaceae. Some of these differences were only present during a certain time, disappearing afterwards. For instance, infants whose mothers received IAP showed lower relative proportions of Bifidobacteriaceae at 10 days of age but not at the age of 1 month, which is in good agreement with the results obtained by Corvaglia and coworkers [46] by using qPCR for the genus Bifidobacterium. Moreover, our results indicate that some IAP-induced microbiota alterations remain for up to 3 months of age. To this regard, Mazzola and coworkers [33] recently reported similar differences to those obtained by us, persisting up to 1 month of age, which was the duration of their study. Similar to our results, Azad et al. [32] have also recently reported reduced levels of Bacteroidetes and increased of Proteobacteria in 3month-old vaginally delivered infants whose mothers received IAP, these differences disappearing by the age of 1 year. Moreover, a similar pattern with increased colonization by potentially pathogenic enterobacteria has also been observed in preterm babies [17]. A question that remains open is the exact mechanism behind the observed effects. The spectrum of action of the antibiotic used, penicillin, may partially explain the observed results since it has a strong activity against gram-positive microorganisms and by reducing them would allow the overgrowth of gram-negatives such as Proteobacteria. However, this can be more complex and depend on the specific spectrum since Firmicutes (gram-positive) also show higher levels in IAP infants whilst the levels of other gram-negative microorganisms, such as those from the phyla Bacteroidetes, are reduced.

The information available on the impact of antibiotic exposure in SCFA production during early life is very scarce. Antibiotic use had been suggested as a potential cause for low fecal SCFA levels [47], and we recently reported lower levels of fecal SCFAs, especially of acetate, in preterm infants exposed to antibiotics [38]. In the same way, in the present study, we observed a trend towards a delayed increase of fecal acetate levels in infants from mothers receiving IAP in comparison with babies not exposed to antibiotics. Given the important roles that the SCFAs play in human physiology [48], this reduced acetate level during the first month of life could have potential effects on infant development and health.

Our results suggest a potential interaction between IAP administration and infant feeding habit. This is especially relevant for the phylum Bacteroidetes whose levels were much lower in formula-fed than in exclusively breast-fed infants. These differences seem to affect the response to IAP that differs between both groups of infants, giving an extra complexity to the understanding of the effects of early-life factors upon the microbiota. To this regard, Mazzola and coworkers [33] recently reported larger effects of IAP in the microbiota of exclusively breast-fed babies than in infants under mixed feeding. On the contrary, other authors found a potential protective role of breast-feeding against the IAP-induced microbiota changes in C-sectiondelivered babies [32]. Different methodological issues such as the limited number of newborns included in these studies, the several potentially confounding factors involved, as well as the challenge of defining infant-feeding regime other than exclusive breast-feeding, have difficulty in drawing firm conclusions. To this regard, our results point out to a differential response to IAP depending on infant feeding which adds more difficulty for reaching general conclusions or making recommendations. However, it would be important to define whether or not a protective role of breastfeeding is actually present since this would provide a rationale for additional efforts to encourage breast-feeding in those cases in which IAP has been used.

Another interesting issue related to the early microbiota composition that has remained largely unexplored is the carriage of antibiotic resistance genes. The increase of multidrug resistant microorganisms is an important concern of public health authorities worldwide. Given the potential role of the gut microbiota as reservoir for antibiotic resistance genes, the early microbiota establishment may also constitute a critical step from this perspective. Some studies have reported the presence of antibiotic resistance genes in the early life microbiota $[49,50]$. Gosalbes and co-workers [50] demonstrated the presence of genes conferring resistance to $\beta$-lactam antibiotics and tetracycline in the meconium of more than half of the newborns. Moreover, the results of these authors suggest the vertical mother-infant transmission of antibiotic resistance genes and underline the potential role of the infant microbiome as a reservoir of resistance genes [50]. Gibson et al. [51] recently reported 
the enrichment of specific antibiotic genes following antibiotic treatments in preterm infants. However, although IAP is a fairly common practice and the most frequent cause of antibiotics exposure to antibiotics in neonates, no study has addressed, until now, the possible impact of this practice on the carriage of antibiotic resistance genes by the infant microbiota. In the present study, we have focused on genes previously reported to be relatively common in infants [49-51] and observed an increased number of infants harboring genes, such as BLA-tem, conferring resistance to the antibiotics used in IAP ( $\beta$-lactam) in the group of newborns whose mothers received IAP. On the contrary, no differences between infants from others receiving and not receiving IAP were observed for genes conferring resistance to antibiotics that are not used in IAP, such as tetracycline. Although the results did not reach statistical significance, likely due to the limited sample size, the observed trend towards an increased carriage of BLA-tem following IAP, with an additional $20 \%$ of infants harboring the gene, deserves further attention and confirmation in larger studies.

Our results, together with those recently reported by other authors, indicate an effect of IAP on the establishing early microbiota. Interestingly, this effect seems to remain for at least the first months of life which represent a very critical time for the correct development of the microbiota-induced host homeostasis [2, 41]. The use of IAP is not a clear-cut decision [52] and is usually recommended in cases in which prenatal screening demonstrates colonization by group B streptococci [53]. Unfortunately, IAP is often also administered in cases in which there is not a clear benefit $[54,55]$ and there is a high rate of administration of inadequate IAP [56]. In general, inappropriate antibiotic use is frequent in the pediatric population, which is a matter of concern [57]. Moreover, it is becoming increasingly evident that antibiotic-induced perturbation in the early microbiota may have profound consequences for later health. Different animal studies have demonstrated that the alteration of the early microbiota with antibiotics increases the risk for autoimmune and metabolic diseases, also affecting behavior [24, 25, 58-60]. Actually, repeated exposure to $\beta$-lactam antibiotics during infancy has been related with an increased weight in later life [61], and the intestinal microbiome of infants has been repeatedly reported to be affected by antibiotic use [21, 62]. All these data point out at the urgent need to rationalize the use of antibiotics and to develop intervention strategies aimed at minimizing the impact of early-life antibiotics exposure on the intestinal microbiota development process.

\section{Conclusions}

This study underlines the effect of maternal IAP on the process of establishment of the intestinal microbiota in the newborn. The initial stages of development may represent the window of opportunity for intestinal microbiota modulation, either towards the establishment of a healthy microbial profile or to an aberrant profile. Thus, it is essential to understand how different perinatal factors, such as the use of IAP, may affect the gut microbiota development in the infant and to develop, if needed, treatments to minimize the impact on the microbiota development of any early-life intervention.

\section{Additional files}

Additional file 1: Figure S1. Rarefaction curves generated for the 165 rRNA sequences obtained from the samples using Chao 1 index $(A)$ and Shannon index (B) (PPTX $192 \mathrm{~kb}$ )

Additional file 2: Figure S2. Box plot of mean alpha diversity obtained by combining the data on infants born from mothers receiving IAP $(n=18)$ or those whose mothers did not receive IAP $(n=22)$ at $2,10,30$, and 90 days of age. (PPTX $57 \mathrm{~kb}$ )

Additional file 3: Table S1. Levels (relative frequencies; \%) of the bacterial families showing differences, in at least one time point analyzed, between infants from mothers receiving IAP and those whose mothers did not receive it. (DOCX $17 \mathrm{~kb}$ )

Additional file 4: Figure S3. Relative proportions (\%) of the main SCFAs (acetate, propionate, and butyrate) in feces from infants whose mothers received intrapartum antimicrobial prophylaxis (IAP) and those whose mothers did not (no IAP). (PPTX $91 \mathrm{~kb}$ )

Additional file 5: Table S2. Relative proportion (\%; mean $\pm \mathrm{sd}$ ) of the five main bacterial phyla in the samples from breast and formula-fed infants either exposed of not to IAP. Asterisk denotes statistically significant differences $(p<0.05)$ between non-IAP- and IAP-exposed infants within the same group. 5Denotes statistically significant differences $(p<0.05)$ between breast-fed and formula-fed infants within the same IAP exposition group. (DOCX $17 \mathrm{~kb}$ )

\section{Acknowledgements}

We show our deepest gratitude to all the families participating in the study.

\section{Funding}

This work was founded by the EU Joint Programming Initiative-A Healthy Diet for a Healthy Life (JPI HDHL, http://www.healthydietforhealthylife.eu/) as well as the Spanish Ministry of Economy and Competitiveness (MINECO) (Project "EarlyMicroHealth" PCIN-2015-233 and Project AGL2013-43770R), and Italian Ministry of Education, University and Research (MIUR) grant to MV and CM. A. Nogacka was the recipient of a FPI contract from MINECO, and NS benefits from a postdoctoral "Juan de la Cierva-Incorporación" grant from MINECO. The Grant GRUPIN14-043 from "Plan Regional de Investigación del Principado de Asturias" is also acknowledged. We acknowledge support of the publication fee by the CSIC Open Access Publication Support Initiative through its Unit of Information Resources for Research (URICI).

\section{Availability of data and materials}

The sequences obtained in this article have been deposited in the NCBI Short Read Archive (SRA) under the project accession number PRJNA362530.

\section{Authors' contributions}

MS, GS, NF, CGR-G, and MG designed research. AN, NS, MS, SA, GS, NF, and MG performed research. $C M, L A, A H-B$, and $M V$ contributed new reagents/analytic tools. AN, NS, CM, MV, and MG analyzed data. CGR-G, MV, and MG wrote the paper. All authors corrected the paper and approved the latest version.

\section{Competing interest}

The authors declare that they have no competing interests.

\section{Ethics approval and consent to participate}

The study was approved by the Regional Ethical Committee of Asturias Public Health Service (SESPA) and an informed written consent was obtained from each infant's parents. 


\section{Consent for publication}

Not applicable.

\section{Publisher's Note}

Springer Nature remains neutral with regard to jurisdictional claims in published maps and institutional affiliations.

\section{Author details}

'Department of Microbiology and Biochemistry of Dairy Products, Instituto de Productos Lácteos de Asturias. Consejo Superior de Investigaciones Científicas (IPLA-CSIC), Ctra. Infiesto s/n, 33300 Villaviciosa, Asturias, Spain. ${ }^{2}$ Pediatrics Service, Hospital Universitario Central de Asturias, SESPA, Oviedo, Asturias, Spain. ' ${ }^{3}$ Laboratory of Probiogenomics, Department of Life Sciences, University of Parma, Parma, Italy. ${ }^{4}$ Current address: APC Microbiome Institute, University College Cork. Cork, Ireland \& Teagasc Food Research Centre, Moorepark, Fermoy, Cork, Ireland.

Received: 3 February 2017 Accepted: 21 July 2017

Published online: 08 August 2017

\section{References}

1. Sekirov I, Russell SL, Antunes LC, Finlay BB. Gut microbiota in health and disease. Physiol Rev. 2010;90:859-904.

2. Sommer F, Bäckhed F. The gut microbiota-masters of host development and physiology. Nat Rev Microbiol. 2013:11:227-38.

3. Cho I, Yamanishi S, Cox L, Methe BA, Zavadil J, Li K, et al. Antibiotics in early life alter the murine colonic microbiome and adiposity. Nature. 2012;488:621-6.

4. Olszak T, An D, Zeissig S, Vera MP, Richter J, Franke A, et al. Microbial exposure during early life has persistent effects on natural killer T cell function. Science. 2012:336:489-93.

5. Cox LM, Yamanishi S, Sohn J, Alekseyenko AV, Leung JM, Cho I, et al. Altering the intestinal microbiota during a critical developmental window has lasting metabolic consequences. Cell. 2014;158:705-21.

6. Gensollen T, Lyer SS, Kasper DL, Blumberg RS. How colonization by microbiota in early life shapes the immune system. Science. 2016;352:539-44.

7. Clarke G, O'Mahony SM, Dinan TG, Cryan JF. Priming for health: gut microbiota acquired in early life regulates physiology, brain and behavior. Acta Paediatr. 2014;103:812-9.

8. Neuman H, Debelius JW, Knight R, Koren O. Microbial endocrinology: the interplay between the microbiota and the endocrine system. FEMS Microbiol Rev. 2015;39:509-21.

9. Kalliomäki M, Collado MC, Salminen S, Isolauri E. Early differences in fecal microbiota composition in children may predict overweight. Am J Clin Nutr. 2008;87:534-8

10. Fujimura KE, Sitarik AR, Havstad S, Lin DL, Levan S, Fadrosh D, et al. Neonatal gut microbiota associates with childhood multisensitized atopy and T cell differentiation. Nat Med. 2016:22:1187-91.

11. Simonyte Sjodin K, Vidman L, Ryden P, West CE. Emerging evidence of the role of gut microbiota in the development of allergic diseases. Curr Opin Allergy Clin Immunol. 2016;16:390-5.

12. Rodriguez JM, Murphy K, Stanton C, Ross RP, Kober Ol, Juge N, et al. The composition of the gut microbiota throughout life, with an emphasis on early life. Microb Ecol Health Dis. 2015;26:26050.

13. Koenig JE, Spor A, Scalfone N, Fricker AD, Stombaugh J, Knight R, et al. Succession of microbial consortia in the developing infant gut microbiome. Proc Natl Acad Sci U S A. 2011;108(Suppl 1):4578-85.

14. Arboleya S, Binetti A, Salazar N, Fernández N, Solís G, Hernández-Barranco A, et al. Establishment and development of intestinal microbiota in preterm neonates. FEMS Microbiol Ecol. 2012;79:763-72.

15. Turroni F, Peano C, Pass DA, Foroni E, Severgnini M, Claesson MJ, et al. Diversity of bifidobacteria within the infant gut microbiota. PLoS One. 2012;7:e36957.

16. Bergström A, Skov TH, Bahl MI, Roager HM, Christensen LB, Ejlerskov KT, et al. Establishment of intestinal microbiota during early life: a longitudinal, explorative study of a large cohort of Danish infants. Appl Environ Microbiol. 2014:80:2889-900.

17. Arboleya S, Sánchez B, Milani C, Duranti S, Solís G, Fernández N, et al. Intestinal microbiota development in preterm neonates and effect of perinatal antibiotics. J Pediatr. 2015;166:538-44.

18. Spor A, Koren O, Ley R. Unravelling the effects of the environment and host genotype on the gut microbiome. Nat Rev Microbiol. 2011;9:279-90.
19. Jakobsson HE, Abrahamsson TR, Jenmalm MC, Harris K, Quince C, Jernberg

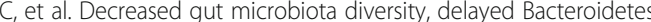
colonization and reduced Th1 responses in infants delivered by caesarean section. Gut. 2014:63:559-66.

20. Bäckhed F, Roswall J, Peng Y, Feng Q, Jia H, Kovatcheva-Datchary P, et al. Dynamics and stabilization of the human gut microbiome during the first year of life. Cell Host Microbe. 2015;17:690-703.

21. Rutten NBMM, Rijkers GT, Meijssen CB, Crijns CE, Oudshoorn JH, van der Ent CK, Vlieger AM. Intestinal microbiota composition after antibiotic treatment in early life: the INCA study. BMC Pediatr. 2015;15:204.

22. Tamburini S, Shen N, Wu HC, Clemente JC. The microbiome in early life: implications for health outcomes. Nat Med. 2016;22:713-22.

23. Bendtsen KM, Fisker L, Hansen AK, Hansen CH, Nielsen DS. The influence of the young microbiome on inflammatory diseases-lessons from animal studies. Birth Defects Res C Embryo Today. 2015:105:278-95.

24. Russell SL, Gold MJ, Hartmann M, Willing BP, Thorson L, Wlodarska M, et al. Early life antibiotic-driven changes in microbiota enhance susceptibility to allergic asthma. EMBO Rep. 2012;13:440-7.

25. Cox LM, Blaser MJ. Antibiotics in early life and obesity. Nat Rev Endocrinol. 2014;1:182-90

26. Nobel YR, Cox LM, Kirigin FF, Bokulich NA, Yamanishi S, Teitler I, et al. Metabolic and metagenomics outcomes from early-life pulsed antibiotic treatment. Nat Commun. 2015;6:7486.

27. Van Dyke MK, Phares CR, Lynfield R, Thomas AR, Arnold KE, Craig AS, et al. Evaluation of universal antenatal screening for group B streptococcus. N Engl J Med. 2009;360:2626-36.

28. Vangay P, Ward T, Gerber JS, Knights D. Antibiotics, pediatric dysbiosis, and disease. Cell Host Microbe. 2015:17:553-64.

29. Bokulich NA, Chung J, Battaglia T, Henderson N, Jay M, Li H, et al. Antibiotics, birth mode, and diet shape microbiome maturation during early life. Sci Transl Med. 2016;8:343ra382.

30. Jaureguy F, Carton M, Panel P, Foncaud P, Butel M-J, Doucet-Populaire F. Effects of intrapartum penicillin prophylaxis on intestinal bacteria colonization in infants. J Clin Microbiol. 2004:42:5184-8.

31. Aloisio I, Quagliariello A, De Fanti S, Luiselli D, De Filippo C, Albanese D, et al. Evaluation of the effects of intrapartum antibiotic prophylaxis on newborn intestinal microbiota using a sequencing approach targeted to multi hypervariable 16S rDNA regions. Appl Microbiol Biotechnol. 2016:100:5537-46.

32. Azad MB, Konya T, Persaud RR, Guttman DS, Chari RS, Field CJ, et al. Impact of maternal intrapartum antibiotics, method of birth and breastfeeding on gut microbiota during the first year of life: a prospective cohort study. BJOG-Int J Obstet Gynecol. 2016;123:983-93.

33. Mazzola G, Murphy K, Ross RP, Di Gioia D, Biavati B, Corvaglia LT, et al. Early gut microbiota perturbations following intrapartum antibiotic prophylaxis to prevent group B streptococcal disease. PLoS One. 2016;11:e0157527.

34. Milani C, Hevia A, Foroni E, Duranti S, Turroni F, Lugli GA, et al. Assessing the fecal microbiota: an optimized ion torrent 165 rRNA gene-based analysis protocol. PLoS One. 2013;8:e68739.

35. Caporaso JG, Kuczynski J, Stombaugh J, Bittinger K, Bushman FD, Costello EK, et al. QIIME allows analysis of high-throughput community sequencing data. Nat Meth. 2010:7:335-6.

36. Edgar RC. Search and clustering orders of magnitude faster than BLAST. Bioinformatics. 2010:26:24601

37. Quast C, Pruesse E, Yilmaz P, Gerken J, Schweer T, Yarza P, et al. The SILVA ribosomal RNA gene database project: improved data processing and webbased tools. Nucl Acids Res. 2013;41:D590-6.

38. Arboleya S, Sánchez B, Solís G, Fernández N, Suárez M, Hernández-Barranco AM, et al. Impact of prematurity and perinatal antibiotics on the developing intestinal microbiota: a functional inference study. Int J Mol Sci. 2016;17:649.

39. Ormerod KL, Wood DLA, Lachner N, Gellaty SL, Daly JN, Parsons JD, et al. Genomic characterization of the uncultured Bacteroidales family S24-7 inhabiting the guts of homeothermic animals. Microbiome. 2016;4:36

40. Lagkouvardos I, Pukall R, Abt B, Foesel BU, Meier-Kolthoff JP, Kumar N, et al. The Mouse Intestinal Bacterial Collection (miBC) provides host-specific insight into cultured diversity and functional potential of the gut microbiota. Nat Microbiol. 2016:1:16131.

41. Renz H, Brandtzaeg P, Hornef M. The impact of perinatal immune development on mucosal homeostasis and chronic inflammation. Nat Rev Imunol. 2012;12:9-23.

42. Faa G, Gerosa C, Fanni D, Nemolato S, van Eyken P, Fanos V. Factors influencing the development of a personal tailored microbiota in the 
neonate, with particular emphasis on antibiotic therapy. J Matern Fetal Neonatal Med. 2013;26(S2):35-43.

43. Dethlefsen L, Relman DA. Incomplete recovery and individualized responses of the human distal gut microbiota to repeated antibiotic perturbation. Proc Natl Acad Sci U S A. 2011;108(Suppl 1):4554-61.

44. Fouhy F, Guinane CM, Hussey S, Wall R, Ryan CA, Dempsey EM, et al. Highthroughput sequencing reveals the incomplete, short-term recovery of infant gut microbiota following parenteral antibiotic treatment with ampicilin and gentamicin. Antimicrob Agents Chemother. 2012;56:5811-20.

45. Avershina E, Storro O, Oien T, Johnsen R, Pope P, Rudi K. Major fecal microbiota shifts in composition and diversity with age in a geographically restricted cohort of mothers and their children. FEMS Microbiol Ecol. 2014;87:280-90.

46. Corvaglia L, Tonti G, Martini S, Aceti A, Mazzola G, Di Gioa D, Faldella G. Influence of intrapartum antibiotic prophylaxis for group B Streptococus on gut microbiota in the first month of life. J Pediatr Gastroenterol Nutr. 2016;62:304-8.

47. Szylit O, Maurage C, Gasqui P, Popot F, Favre A, Gold F, et al. Fecal shortchain fatty acids predict digestive disorders in premature infants. JPEN J Parenter Enteral Nutr. 1998;22:136-41.

48. Ríos-Covián D, Ruas-Madiedo P, Margolles A, Gueimonde M, de Los ReyesGavilán CG, Salazar N. Intestinal short chain fatty acids and their link with diet and human health. Front Microbiol. 2016;7:185.

49. Gueimonde M, Salminen S, Isolauri E. Presence of specific antibiotic (tet) resistance genes in infant faecal microbiota. FEMS Immunol Med Microbiol. 2006;48:21-5.

50. Gosalbes MJ, Valles Y, Jimenez-Hernández M, Balle C, Riva P, Miravet-Verde $S$, et al. High frequencies of antibiotic resistance genes in infants's meconium and early fecal samples. J Dev Orig Health Dis. 2016;7:35-44.

51. Gibson MK, Crofts TS, Dantas G. Antibiotics and the developing infant gut microbiota and resistome. Curr Opin Microbiol. 2015;27:51-6.

52. Kenyon S, Boulvain M, Neilson JP. Antibiotics for preterm rupture of membranes. Cochrane Database Syst Rev. 2010;8:CD001058.

53. Allen VM, Yudin MH, Bouchard C, Boucher M, Caddy S, Castillo E, et al. Management of group B streptococcal bacteriuria in pregnancy. J Obstet Gynaecol Can. 2012;34:482-6.

54. Flenady V, King J. Antibiotics for prelabour rupture of membranes at or near term. Cochrane Database Syst Rev. 2002;3:CD001807.

55. King JF, Flenady V, Murray L. Prophylactic antibiotics for inhibiting preterm labour with intact membranes. Cochrane Database Syst Rev. 2002:4:CD000246

56. Bienenfeld S, Rodríguez-Riesco LG, Heyborne KD. Avoiding inadequate intrapartum prophylaxix for group B streptococci. Obstet Gynecol. 2016; 128:598-603.

57. Bedford Russell AR, Murch SH. Could peripartum antibiotics have delayed health consequences for the infant? BJOG-Int J Obstet Gynecol. 2006;113:758-65.

58. Candon S, Perez-Arroyo A, Marquet C, Valette F, Foray A-P, Pelletier B, et al. Antibiotics in early life alter the gut microbiome and increase disease incidence in a spontaneous model of autoimmune insulin-dependent diabetes. PLoS One. 2015;10:e0125448.

59. Livanos AE, Greiner TU, Vangay P, Pathmasiri W, Steward D, McRitchie S, et al. Antibiotic-mediated gut microbiome perturbation accelerates development of type 1 diabetes in mice. Nat Microbiol. 2016;1:16140.

60. Tochitani S, Ikeno T, Ito T, Sakurai A, Yamauchi T, Matsuzaki H. Administration of non-absorbable antibiotics to pregnant mice to perturb the maternal gut microbiota is associated with alterations in offspring behavior. PLoS One. 2016;1:e0138293.

61. Mbakwa CA, Scheres L, Penders J, Mommers M, Thiij C, Arts ICW. Early life antibiotic exposure and weight development in children. J Pediatr. 2016;176:105-13.

62. Korpela K, Salonen A, Virta LJ, Kekkonen RA, Forslund K, Bork P, de Vos WM. Intestinal microbiome is related to lifetime antibiotic use in Finnish preschool children. Nat Commun. 2016;7:10410.

63. Bailey JK, Pinyon JL, Anantham S, Hall RM. Commensal Escherichia coli of healthy humans: a reservois for antibiotic-resistance determinants. J Med Microbiol. 2010;59:1331-9.

64. Fouhy F, Ross RP, Fitzgerald GF, Stanton C, Cotter PD. A degenerate PCR-based strategy as a means of identifying homologues of aminoglycoside and $\beta$-lactam resistance genes in the gut microbiota. BMC Microbiol. 2014;14:25.

\section{Submit your next manuscript to BioMed Central and we will help you at every step:}

- We accept pre-submission inquiries

- Our selector tool helps you to find the most relevant journal

- We provide round the clock customer support

- Convenient online submission

- Thorough peer review

- Inclusion in PubMed and all major indexing services

- Maximum visibility for your research

Submit your manuscript at www.biomedcentral.com/submit

Biomed Central 